Check for updates

Cite this: RSC Adv., 2018, 8, 18952

Received 16th February 2018

Accepted 14th May 2018

DOI: $10.1039 / c 8 r a 01466 c$

rsc.li/rsc-advances

\section{Binding studies between cytosinpeptidemycin and the superfamily 1 helicase protein of tobacco mosaic virus}

\author{
Xiangyang Li, D * Kai Chen, Di Gao, Dongmei Wang, Maoxi Huang, Hengmin Zhu \\ and Jinxin Kang
}

Tobacco mosaic virus (TMV) helicases play important roles in viral multiplication and interactions with host organisms. They can also be targeted by antiviral agents. Cytosinpeptidemycin has a good control effect against TMV. However, the mechanism of action is unclear. In this study, we expressed and purified TMV superfamily 1 helicase (TMV-Hel) and analyzed its three-dimensional structure. Furthermore, the binding interactions of TMV-Hel and cytosinpeptidemycin were studied. Microscale thermophoresis and isothermal titration calorimetry experiments showed that cytosinpeptidemycin bound to TMV-Hel with a dissociation constant of $0.24-0.44 \mu \mathrm{M}$. Docking studies provided further insights into the interaction of cytosinpeptidemycin with the His375 of TMV-Hel. Mutational and Microscale thermophoresis analyses showed that cytosinpeptidemycin bound to a TMV-Hel mutant ( $\mathrm{H} 375 \mathrm{~A})$ with a dissociation constant of $14.5 \mu \mathrm{M}$. Thus, His375 may be the important binding site for cytosinpeptidemycin. The data are important for designing and synthesizing new effective antiphytoviral agents.

\section{Introduction}

Superfamily 1 (SF1) helicases are encoded in the small and large subunits of tobacco mosaic virus (TMV) and tomato mosaic virus (ToMV). ${ }^{1}$ They play important roles in viral replication. After infection, the genomic RNA is translated to produce the SF1 helicase in the cytoplasm of the host cell. ${ }^{2}$ During viral-RNA replication and control of host defenses, the SF1 helicase interacts with itself ${ }^{3}$ and with host proteins, such as TOM1, ARL8 and auxin/indole-3-acetic acid-related proteins. ${ }^{4-8}$ Then, the resistance genes $\mathrm{N}^{9}$ and $\mathrm{Tm}-1$ (ref. 10 and 11) are produced and bind replication proteins to inhibit replication complex formation. ${ }^{12-14}$

The ToMV SF1 helicases' (ToMV-Hel) structure, which contains two RecA domains and an N-terminal accessory domain, was determined using X-ray crystallography. ${ }^{15}$ In the ToMV-Hel N-terminal accessory domain, a long $\alpha$-helix binds to TOM1 and ARL8 (ref. 4-6) for viral replication. The primary amino acid sequence of TMV helicases (TMV-Hel) have high levels of similarity to that of ToMV-Hel. The TMV-Hel and ToMV-Hel belong to Tobamovirus. ${ }^{16}$ The TMV-Hel and ToMVHel are also highly similar in secondary structure and function. ${ }^{17}$ Therefore, TMV-Hel is an important protein and may be a target for antiphytoviral compounds.

State Key Laboratory Breeding Base of Green Pesticide and Agricultural Bioengineering, Key Laboratory of Green Pesticide and Agricultural Bioengineering, Ministry of Education, Guizhou University, Guiyang 550025, PR China. E-mail: xyli1@gzu.edu.cn; Fax: +86-851-88292090; Tel: +86-851-88292090
Cytosinpeptidemycin is an antiphytoviral antibiotic. Zhu et al. (2005) reported that cytosinpeptidemycin showed an $82.6 \%$ protection activity and $95.3 \%$ inactivate activity against TMV in tobacco. Zhu et al., using $3 \mathrm{H}$-uridine and $3 \mathrm{H}$ leucine tracing, found that the mechanism of cytosinpeptidemycin against TMV involved the synthesis of TMV RNA. ${ }^{\mathbf{1 8 , 1 9}}$ Li et al. reported that cytosinpeptidemycin could inhibit the synthesis of the TMV methyltransferase-guanylyltransferaselike domain (at the $\mathrm{N}$-terminus) and helicase-like domain (at the C-terminus) proteins using western blotting of BY-2 tobacco protoplasts. Furthermore, cytosinpeptidemycin could inhibit TMV RNA synthesis as shown in northern blots. ${ }^{20}$ However, the site of action on TMV RNA by cytosinpeptidemycin is unclear.

Because of its importance in TMV-associated disease transmission, TMV-Hel was expressed and regarded as a potential target for cytosinpeptidemycin. Here, we report binding studies using cytosinpeptidemycin and the TMV-Hel protein. Microscale thermophoresis (MST) studies revealed that the binding affinity of cytosinpeptidemycin for the TMV-Hel protein was strong, with a dissociation constant of $0.44 \mu \mathrm{M}$. Molecular dynamics and mutagenesis studies revealed that His375 in TMV-Hel was an important binding site for cytosinpeptidemycin. Our results demonstrated that cytosinpeptidemycin had an affinity for the TMV-Hel protein through a direct interaction with residue His375. To the best of our knowledge, this is the first report explaining the mechanism of cytosinpeptidemycin, which shows an antiviral activity against TMV. 


\section{Materials and methods}

\subsection{Drug and virus}

Cytosinpeptidemycin (30\%), original agent, was bought from an insecticide factory. The structural formula of compound is list in Table 1 . Tobacco mosaic virus was preserved in tobacco $\mathrm{K}_{326}$ in our laboratory.

\subsection{RNA extraction, RT-PCR and plasmid constructs}

TMV total RNA was extracted using the RNA TRIzol reagent (TaKaRa, Japan), and the total RNA was detected using a Genequant100 (GE Healthcare, USA). The cDNA synthesis methods were described in a previous report. ${ }^{21}$ The gene sequence (GenBank accession no. AJ011933.1) for TMV-Hel was used to design primers containing a Not I (underlined) restriction site in the forward primer (5'-ATAAGAATGCGGCCGCATGTCCTATTCTAAG- $\left.3^{\prime}\right)$ and a XhoI (underlined) site in the reverse primer (5'-CCGCTCGAGTTAATCGACCTTATACATATC- $3^{\prime}$ ). The full-length $T M V-H e l$ gene was inserted into the vector pMCSG19 (Novagen, USA), resulting in the prokaryotic expression plasmid PMCSG19-TMV-Hel. The TMV-Hel mutant (H375A) was cloned and confirmed by $1 \%$ agarose gel electrophoresis and DNA sequencing (Shanghai Sangon Company, Shanghai, China).

\subsection{Protein expression and purification}

A freshly transformed overnight culture of Escherichia coli strain Rosetta(DE3) (Novagen, USA) containing the plasmid pMCSG19-TMV-Hel was transferred to $1 \mathrm{~L}$ Luria broth supplemented with $50 \mu \mathrm{g} \mathrm{mL}{ }^{-1}$ ampicillin at $37{ }^{\circ} \mathrm{C}$ until the $\mathrm{OD}_{600}$ reached 0.6-1.0. Then, protein expression was induced with $0.8 \mathrm{mM}$ isopropyl- $\beta$-D-galactopyranoside at $16{ }^{\circ} \mathrm{C}$ overnight. The cells were removed, immediately placed in centrifuge tubes and immersed in liquid nitrogen. The cells were harvested, resuspended in lysis buffer (50 mM Tris- $\mathrm{HCl}, 150 \mathrm{mM} \mathrm{NaCl}$ and $1 \mathrm{mM} \beta$-mercaptoethanol, $\mathrm{pH}$ 7.5) and then lysed at $4{ }^{\circ} \mathrm{C}$ by sonication. ${ }^{22}$ The lysate was clarified by centrifugation at $12000 \mathrm{~g}$ for $30 \mathrm{~min}$ at $4{ }^{\circ} \mathrm{C}$, the soluble supernatant was loaded onto a $5 \mathrm{~mL}$ His-Trap high-performance column (GE Healthcare), and the protein was eluted with a linear gradient of 50$400 \mathrm{mM}$ imidazole ( $\mathrm{pH}$ 7.5). Eluate fractions collected from the column were analyzed by $12 \%$ SDS-PAGE, and the crude protein was isolated at $4{ }^{\circ} \mathrm{C}$ using a desalting column (GE Healthcare) attached to an AKTA purifier protein liquid chromatography system (GE Healthcare). The fractions containing target proteins were pooled, concentrated to a suitable concentration by ultrafiltration (10 kDa cut-off), and the MBP-tag was digested by TEV protease at $4{ }^{\circ} \mathrm{C}(1 \mathrm{mg}$ TEV protease with $100 \mathrm{mg}$ MBPtagged fusion protein). The protein concentration was determined using a Genequant100 system (GE Healthcare), and the protein was stored at $-80{ }^{\circ} \mathrm{C}$ for further analyses.

\subsection{Size-exclusion chromatography (SEC) and PAGE analysis}

The purified proteins were loaded onto the Superdex 200 10/300 GL column (GE Healthcare) of an AKTA purifier protein liquid chromatography system (GE Healthcare), and the peak protein states were estimated by comparisons with protein standards. The molecular mass standards (Bio-Rad, USA) included thyroglobulin (669 kDa), ferritin (440 kDa), bovine serum albumin (67 kDa), $\beta$-lactoglobulin (35 kDa), ribonuclease A (13.7 kDa), cytochrome $(13.6 \mathrm{kDa})$, aprotinin $(6.51 \mathrm{kDa})$, and vitamin B12 $(1.36 \mathrm{kDa})$. The proteins were monitored by absorbance at $280 \mathrm{~nm}$.

The fractions under the peak were collected and analyzed by $12 \%$ SDS-PAGE at room temperature. Briefly, $20 \mu \mathrm{L}$ sample was mixed with $20 \mu \mathrm{L} 2 \times$ loading buffer [100 mM Tris-HCl (pH 6.8), $200 \mathrm{mM}$ DTT, $4 \%$ (m/v) SDS, $0.2 \%$ bromophenol blue (w/v), and $20 \%$ glycerin $(\mathrm{v} / \mathrm{v})]$. Subsequently, $4 \mu \mathrm{L}$ samples were loaded onto $12 \%$ gels. Electrophoresis was performed using a $1 \times$ SDSPAGE buffer [ $25 \mathrm{mM}$ Tris, $250 \mathrm{mM}$ glycine $(\mathrm{pH} 8.3$ ), and $0.1 \%$ SDS $(\mathrm{m} / \mathrm{v})$ ] initially at $150 \mathrm{~V}$ at $22{ }^{\circ} \mathrm{C}$ for $20 \mathrm{~min}$, and then at $120 \mathrm{~V}$ at $22{ }^{\circ} \mathrm{C}$ for $55 \mathrm{~min}$. After electrophoresis, gels were stained with Coomassie Brilliant Blue to identify proteins, and then destained with methanol and glacial acetic acid.

\subsection{Homologous model of TMV-Hel}

The homology of the complete nucleotide sequences TMV-Hel and ToMV-Hel (S666 to Q1116) was analyzed using the EMBLEBI sequence alignment program server (http:/www.ebi.ac.uk/ Tools/msa/clustalw2/). The secondary structures of TMV-Hel were predicted based on their primary amino acid sequences using the ExPASy structural program server (http:// www.expasy.org/structural_bioinformatics). The structure of TMV-Hel used for the study was modeled using the homologous structure of ToMV-Hel from PDB (PDB ID: 3VKW) using the ExPASy structural information for the protein (http:// www.expasy.org/structural_bioinformatics). The structural representations of TMV-Hel were generated using pyMOL software (http://www.pymol.org/). ${ }^{23}$

Table 1 Tobacco mosaic virus was preserved in tobacco $\mathrm{K}_{326}$ in our laboratory

Drugs name

Cytosinpeptidemycin
Molecular formula<smiles>NC(=O)C(CO)C(C=O)CNC(CO)C(=O)NCOC(O)C(O)C(O)Cn1ccc(N)nc1=O</smiles> 


\subsection{Binding analysis between cytosinpeptidemycin and TMV-Hel}

The binding was calculated using MST on a Monolith NR 115 (Nano Temper Technologies, Germany). Required compounds at ranging from $0.1 \mathrm{mM}$ to $2 \mathrm{mM}$ were incubated with $0.1 \mathrm{mM}$ of purified recombinant protein for $5 \mathrm{~min}$ with the Monolith NT Protein Labeling Kit Red (Nano Temper Technologies) in assay buffer (10 mM PB and $150 \mathrm{mM}$ sodium chloride, $\mathrm{pH}$ 7.5). The sample was loaded into the NanoTemper glass capillaries and microthermophoresis was carried out using 40\% LED power and $40 \%$ MST. The $K_{\mathrm{d}}$ values were calculated using the mass action equation with the NanoTemper software from duplicate reads of triplicate experiments. Data are performed presented as means \pm SDs of triplicate assays. Statistical differences among the data were evaluated using NanoTemper software. ${ }^{\mathbf{2 4 , 2 5}}$ Isothermal titration calorimetry (ITC)-binding experiments were performed using the ITC 200 Micro Calorimeter (GE Healthcare) at $25^{\circ} \mathrm{C}$. The buffer contained $10 \mathrm{mM}$ Tris- $\mathrm{HCl}$ and $150 \mathrm{mM}$ sodium chloride ( $\mathrm{pH} 7.5)$. The compounds ( $2 \mathrm{mM}$ ) were titrated into TMV-Hel $(0.1 \mathrm{mM})$ in a $200 \mu \mathrm{L}$ sample cell using a $40 \mu \mathrm{L}$ microsyringe, as follows: $0.4 \mu \mathrm{L}$ for the first injection and $2 \mu \mathrm{L}$ for the next 19 injections at intervals of $150 \mathrm{~s}$. The integrated heat data were analyzed using the one-set-of-sites model in MicroCal Origin 7.0 according to the manufacturer's instructions. Data are presented as means \pm SDs of triplicate assays. Statistical differences among the data were evaluated using MicroCal Origin 7.0 software. The first data point was not used in the analysis. The binding parameters reaction enthalpy changes in cal mol${ }^{-1}(\Delta H)$, binding constant in $\mathrm{mol}^{-1}(K)$ and number of molecules per TMV-Hel $(n)$ were floating during the fit. The binding free energy $(\Delta G)$ and reaction entropy $(\Delta S)$ were calculated using the following equations:

$$
\Delta G=-R T \ln K\left(R=1.9872 \mathrm{cal} \mathrm{mol}^{-1} \mathrm{~K}^{-1}, T=298 \mathrm{~K}\right),
$$

and

$$
\Delta G=\Delta H-T \Delta S
$$

The dissociation constant $K_{\mathrm{d}}$ was calculated as $1 / K^{26}$

\subsection{Molecular docking of cytosinpeptidemycin and TMV-}

Hel

The initial structure of TMV-Hel was revised by adding lost residues and hydrogen atoms, and by confirming bonds and bumps. Subsequently, energy was minimized using the steepest descent calculations and conjugated gradient calculations using Discovery studio 4.5 (Accelrys, USA). ${ }^{27}$ Optimized geometries were used to construct the entire structures. The final structures were optimized by fixing the macrocycle with a conjugated gradient.

The atomic Gasteiger-Huckel charges were assigned to the ligand and receptor. Most of the parameters for the docking calculation were set to the default values recommended by the software. Each docked structure was scored by the built-in scoring function and was clustered by $0.8 \AA$ of RMSD criteria. $^{28}$ For each binding model, a molecular mechanics/ Poisson-Boltzmann surface area analysis was performed. Before these calculations were made, the complex structure was further refined initially with the steepest descent algorithm and then the conjugated gradient algorithm using the Amber9 package. ${ }^{29}$ During the energy minimization process, the receptor was first fixed and only the ligand remained free. Then, the ligand and residue side chains remained free. Finally, all atoms of the system were liberated and refined to a convergence of $0.01 \mathrm{kcal}\left(\mathrm{mol}^{-1} \AA^{-1}\right)$.

\subsection{Verifying the interaction between cytosinpeptidemycin and the mutated TMV-Hel}

To verify the results of the molecular docking results, a mutation, H375A, was introduced into TMV-Hel. The mutant proteins were used to measure the interactions between cytosinpeptidemycin and TMV-Hel, as previously described..$^{\mathbf{2 4 - 2 6}}$

\section{Results and discussion}

\subsection{Molecular cloning and sequence analysis of TMV-Hel}

The TMV-Hel protein was cloned into pMCSG19 and highly expressed in E. coli. The TMV-Hel protein from the TMV replication protein's S1 to Q451 segment (corresponding to S666 to Q1116) was successfully amplified using specific primers and cloned into the pMCSG19 vector (Fig. 1). A sequence analysis showed that TMV-Hel (isolated from Guizhou Province) consisted of $1353 \mathrm{nt}$ and shared a 99\% nucleotide identity with the published sequence of a TMV replication protein isolates in National Center of Biotechnology Information protein database (GenBank accession no. AJ011933.1).

\subsection{Expression and purification of TMV-Hel}

To obtain sufficient quantities of TMV-Hel for biochemical analyses, the expression vector pMCSG19 containing the TMV$\mathrm{Hel}$ gene was transformed into E. coli strain Rosetta (DE3). A large number of $\mathrm{TMV}-\mathrm{Hel}$ genes were overexpressed when the final isopropyl- $\beta$-D-galactopyranoside concentration was increased to $0.8 \mathrm{mM}$ and the solution was left overnight at $16{ }^{\circ} \mathrm{C}$

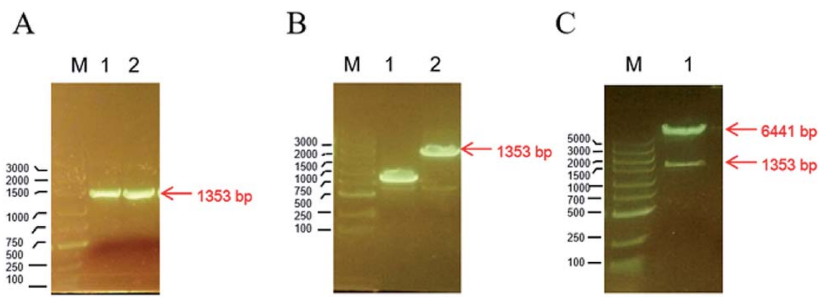

Fig. 1 Construction of TMV-Hel in pMCSG19 plasmid. (A) The PCRamplified and $1 \%$ agarose gel electrophoresis results showed that the length of TMV-Hel segments was $1353 \mathrm{bp}, \mathrm{M}$ was the DNA marker, 1 and 2 was two repeats. (B) $M$ was marker, 1 was the false positive monoclonal of PMCSG19-TMV-Hel, 2 was the positive monoclonal of pMCSG19-TMV-Hel with 1353 bp TMV-Hel segments in pMCSG19. (C) The verification of pMCSG19-TMV-Hel by restriction enzyme Not I and Xho I, M is marker, 1 was the pMCSG19 segments with $6441 \mathrm{bp}$ and TMV-Hel segments with $1353 \mathrm{bp}$. 
(Fig. 2A and B). The His-MBP-tagged fusion protein was purified using a Ni-NTA column (GE Healthcare). More than $90 \%$ of the TMV-Hel-MBP protein was eluted from the beads with $400 \mathrm{mM}$ imidazole in Tris-HCl buffer ( $\mathrm{pH} 7.5)$, and an $89 \mathrm{kDa}$ protein was revealed by $12 \%$ SDS-PAGE analysis (Fig. 2C). The final yield from $500 \mathrm{~mL}$ of bacterial culture was $\sim 0.5 \mathrm{mg}$ of purified TMVHel-MBP protein after using a desalting column. Then, MBP tags were digested by TEV protease to produce TMV-Hel protein.

\subsection{The aggregating state of TMV-Hel}

To determine the aggregating state of TMV-Hel in vitro, fresh TMV-Hel was isolated and analyzed by size-exclusion chromatograph. The TMV-Hel eluted in the corresponding position of $67 \mathrm{kDa}$ in $10 \mathrm{mM}$ Tris- $\mathrm{HCl}$ and $150 \mathrm{mM}$ sodium chloride $(\mathrm{pH}$ 7.5) (Fig. 2D). Thus, the monomer form of TMV-Hel was $\sim 49$ $\mathrm{kDa}$.

\subsection{Interactions between cytosinpeptidemycin and TMV-}

Hel

The binding interactions between cytosinpeptidemycin and TMV-Hel were analyzed by MST. Cytosinpeptidemycin bound to TMV-Hel protein with a $K_{\mathrm{d}}$ of $0.44 \pm 0.16 \mu \mathrm{M}$ (Fig. 3A). Analysis by ITC revealed that one TMV-Hel interacted with three cytosinpeptidemycin molecules, and cytosinpeptidemycin bound to TMV-Hel with a $K$ of $4.16 \pm 2.23 \times 10^{6} \mathrm{M}^{-1}$ (Fig. 3B). The titration data also indicated an apparent negative enthalpy value $\left(\Delta G=-1.74 \times 10^{7} \mathrm{~kJ} \mathrm{~mol}^{-1}\right)$ during the binding of
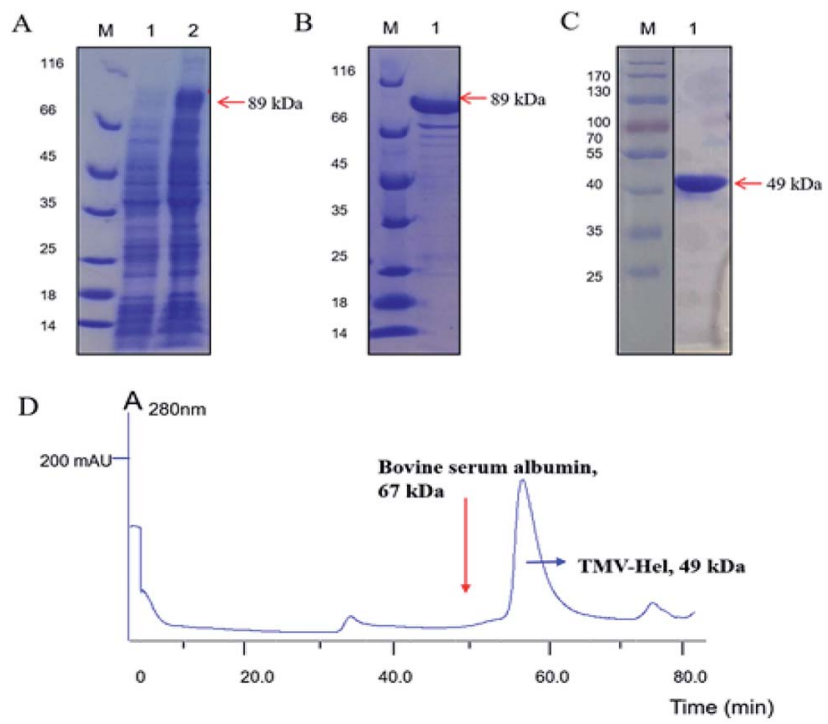

Fig. 2 Purification and characterization of TMV-Hel. (A) TMV-Hel protein with MBP Tag was determined by $12 \%$ SDS-PAGE analysis with $89 \mathrm{kDa}$ in lane 2, M was the protein marker, 1 was control. (B) TMV-Hel protein with MBP Tag was purified in lane 1 by Ni-NT affinity chromatography, $M$ was the protein marker. (C) TMV-Hel protein with MBP Tag was digested by TEV protease to product TMV-Hel protein by $12 \%$ SDS-PAGE analysis with $49 \mathrm{kDa}$ in lane 1, M was the protein marker. (D) TMV-Hel protein eluted under the corresponding position of $67 \mathrm{kDa}$ in $10 \mathrm{mM}$ Tris/ $\mathrm{HCl}, 150 \mathrm{mM}$ sodium chloride (pH 7.5) by using SEC analysis with about $49 \mathrm{kDa}$.
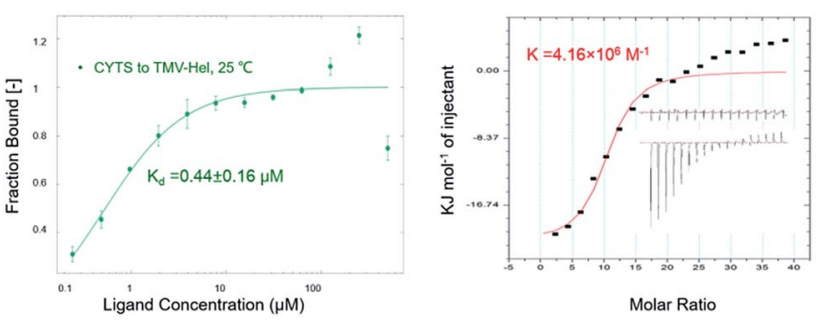

Fig. 3 Interactions between cytosinpeptidemycin and TMV-Hel measured by MST and ITC methods. (A) MST measurements showed that cytosinpeptidemycin bound to TMV-Hel with a dissociation constant $\left(K_{\mathrm{d}}\right)$ of $0.44 \mu \mathrm{M}$. (B) ITC results showed that one TMV-Hel combined three cytosinpeptidemycin molecule with a binding affinity (K) of $4.16 \times 10^{6} \mathrm{M}^{-1}$, and its corresponding $K_{d}$ is $0.24 \mu \mathrm{M}$, which similar to MST measurements.

cytosinpeptidemycin to TMV-Hel. Cytosinpeptidemycin had a micromole affinity with TMV-Hel.

\subsection{Homology modelling of TMV-Hel}

A BLAST algorithm-based analysis using the EMBL-EBI sequence alignment program server, found an $89.6 \%$ homology between TMV-Hel and ToMV-Hel. Based on their primary amino acid sequences, the TMV-Hel protein's secondary structures were predicted, as shown in Fig. 4. The homology structure of TMV-Hel was modelled using the X-ray crystal structure of ToMV-Hel (PDB ID: 3VKW). The structural representation of TMV helicase is shown in Fig. 5.

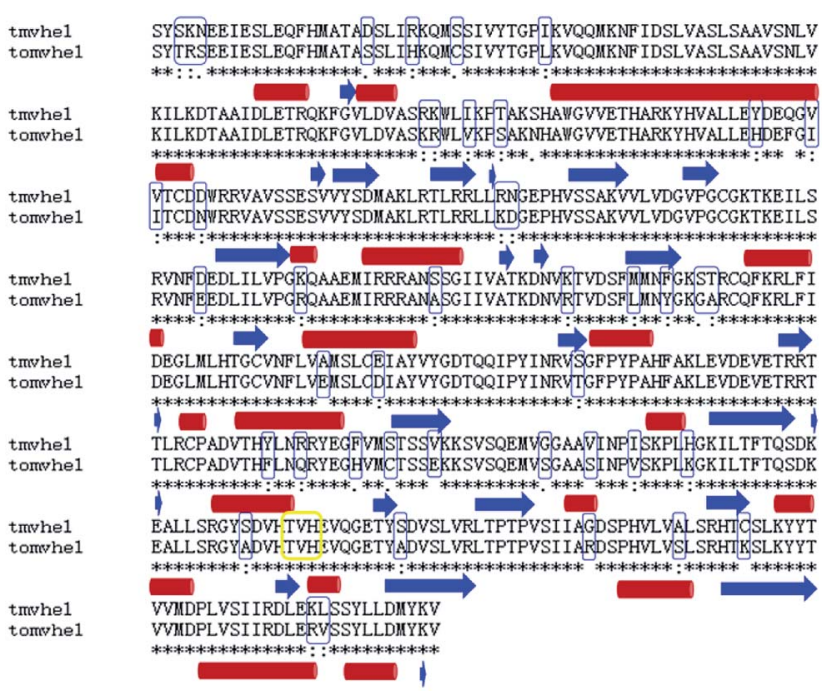

Fig. 4 Alignment of the amino acid sequences of TMV-Hel and ToMVHel. Secondary structural elements were indicated schematically under the X-ray crystal structure of ToMV-Hel. There was $89.6 \%$ homology between TMV-Hel and ToMV-Hel, the residue His375 has highly conserved regions in both TMV-Hel and ToMV-Hel. Secondary structural elements were indicated schematically under the sequences, generated by EMBL-EBI sequence alignment programs server, and indicated as difference residues (blue box) or red cylinder ( $\alpha$ helices) or green arrows ( $\beta$ sheets), the nucleic acid binding motifs of TMV-Hel was labelled by yellow box. 
A

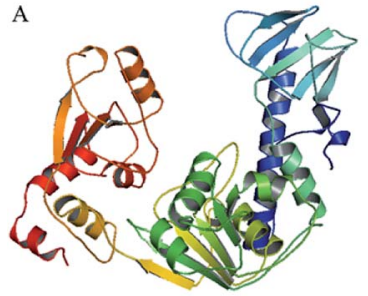

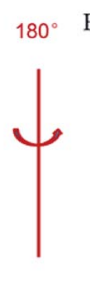

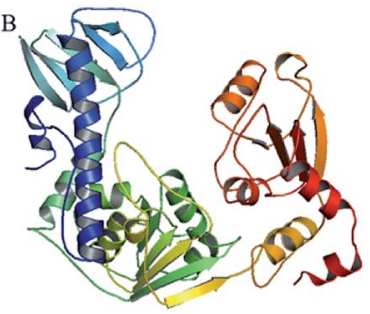

Fig. 5 Based on the X-ray crystal structure of ToMV-Hel (PDB code: 3VKW), the TMV-Hel was modeled. (A) TMV-Hel; (B) was rotate 180 degrees of (A). The figures for structural representation of TMV-Hel were generated on pyMOL software.

\subsection{Molecular docking of cytosinpeptidemycin and TMV-} Hel

To identify the cytosinpeptidemycin recognition sites in TMVHel, we performed molecular docking using the Gold method with 200 cycles. After optimization, we found that His375 in the TMV-Hel shared two hydrogen bonds with cytosinpeptidemycin, $\mathrm{C}=\mathrm{O} \cdots \mathrm{H}-\mathrm{N}=3.12 \AA$ and $\mathrm{H}-\mathrm{N} \cdots \mathrm{H}-\mathrm{N}=3.73 \AA$ (Fig. 6). The BLAST algorithm-based analysis revealed that TMV-Hel shared a high homology level with known ToMV-Hel protein in GenBank, with His375 being present in a highly conserved region (Fig. 4). His375 (named His1040 in ToMV-Hel) is an important residue for TMV-Hel nucleic acid binding. ${ }^{30}$ These analyses indicated that His375 is the reason for the decreasing activity level of cytosinpeptidemycin.

\subsection{Mutational analysis of the protein-ligand interactions between cytosinpeptidemycin and TMV-Hel}

To analyze the roles of the critical residue in the binding of TMV-Hel by cytosinpeptidemycin that was identified in the simulations, we cloned and expressed a TMV-Hel mutant, H375A. The interactions between the mutant and cytosinpeptidemycin were studied using MST, the affinity level between the TMV-Hel mutant and cytosinpeptidemycin decreased $(14.5 \pm 2.05 \mu \mathrm{M})$ compared with the wild type $(0.44 \pm$ $0.16 \mu \mathrm{M}$ ) (Fig. 7). Thus, we deduced that His375 is the binding site of TMV-Hel.

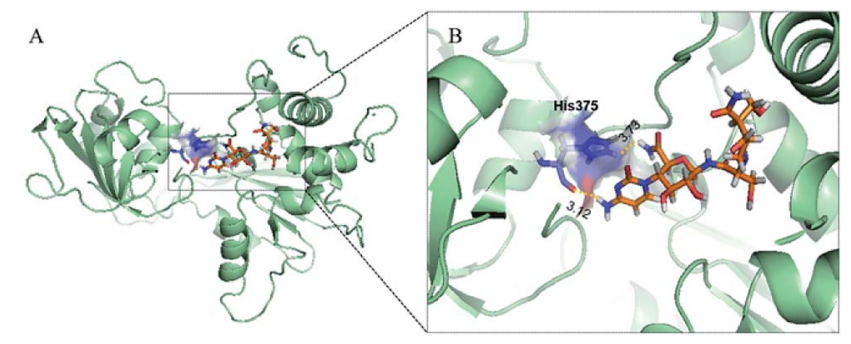

Fig. 6 Docking analysis of the interactions between cytosinpeptidemycin and TMV-Hel. (A) The binding-sites between cytosinpeptidemycin and TMV-Hel by using Discovery studio 4.5; (B) was a partial enlarged detail of $(A)$, two binding-sites, $\mathrm{C}=\mathrm{O} \cdots \mathrm{H}-\mathrm{N}=3.12 \AA$ and $\mathrm{H}-$ $\mathrm{N} \cdots \mathrm{H}-\mathrm{N}=3.73 \AA$, were found in the representative conformation. The stick model stands for cytosinpeptidemycin and His375.

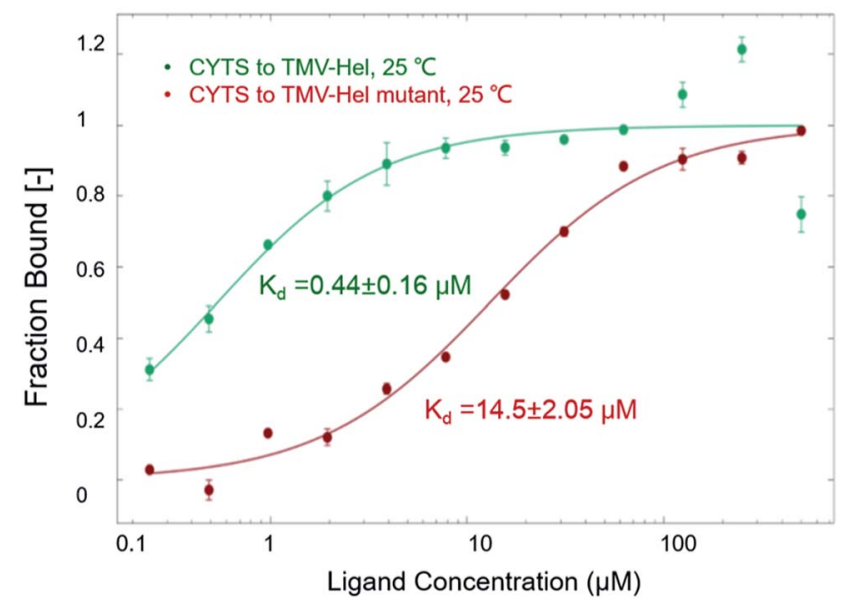

Fig. 7 MST studies of cytosinpeptidemycin and TMV-Hel mutant. MST results showed that the $K_{d}$ of cytosinpeptidemycin and TMV-Hel mutant ( $\mathrm{H} 375 \mathrm{~A})$ was $14.5 \mu \mathrm{M}$. It was 32 times of the $K_{d}$ of cytosinpeptidemycin and TMV-Hel.

\subsection{The putative reaction mechanism of cytosinpeptidemycin on TMV-Hel}

Recent reports showed that the ToMV-Hel's conserved helicase motifs had NTP and nucleic acid-binding sites. ${ }^{31-35}$ The high homology of TMV-Hel with ToMV-Hel indicates that they may have similar functions. Furthermore, cytosinpeptidemycin could inhibit the synthesis of TMV-Hel as shown by western blotting in BY-2 tobacco protoplasts, ${ }^{20}$ and TMV-Hel's His375 is associated with RNA binding. ${ }^{30}$ The residue His375 was contained in a highly conserved region in both TMV-Hel and ToMV$\mathrm{Hel}$; therefore, the biological function of His375 in TMV-Hel could also be associated with RNA binding. Our experiments demonstrated that His375 was also the binding site of cytosinpeptidemycin. Based on these results, we hypothesize that cytosinpeptidemycin inhibited the virulence of TMV through its interaction with His375.

\section{Conclusions}

In summary, our study aimed to explain the mechanism of action of cytosinpeptidemycin against TMV-Hel. First, we expressed TMV-Hel and constructed a structural model as a potential target for antiphytoviral compounds. Second, we found that cytosinpeptidemycin had a micromole affinity for TMV-Hel. Last, we confirmed that cytosinpeptidemycin bound His375 in the TMV-Hel protein, which was important for the binding of TMV RNA. Thus, cytosinpeptidemycin inhibited the virulence of TMV in vitro by binding TMV-Hel via chemical bonds. This competition for the residues contributed to viral replication. Our results indicate, for the first time, that cytosinpeptidemycin inhibits TMV infection by binding His375 in the TMV-Hel protein.

To the best of our knowledge, this is the first reported explanation of the mechanism for cytosinpeptidemycin that shows antiviral activity against TMV-Hel, and this study also 
establishes a useful model system that allows the rapid screening of antiviral agents.

\section{Conflicts of interest}

There are no conflicts to declare.

\section{Acknowledgements}

This study was supported by the National Natural Science Foundation of China (No. 21502032 and No. 31460460).

\section{References}

1 G. Kadare and A. L. Haenni, Virus-encoded RNA helicases, J. Virol., 1997, 71, 2583-2590.

2 S. P. Goregaoker, D. J. Lewandowski and J. N. Culver, Identification and functional analysis of an interaction between domains of the $126 / 183-\mathrm{kDa}$ replicase-associated proteins of tobacco mosaic virus, Virology, 2001, 282, 320328.

3 K. Ishibashi, M. Nishikiori and M. Ishikawa, Interactions between tobamovirus replication proteins and cellular factors: their impacts on virus multiplication, Mol. PlantMicrobe Interact., 2010, 23, 1413-1419.

4 M. Nishikiori, M. Mori, K. Dohi, H. Okamura, E. Katoh, S. Naito, T. Meshi and M. Ishikawa, A host small GTPbinding protein ARL8 plays crucial roles in Tobamovirus RNA replication, PLoS Pathog., 2011, 7, e1002409.

5 T. Yamanaka, T. Imai, R. Satoh, A. Kawashima, M. Takahashi, K. Tomita, K. Kubota, T. Meshi, S. Naito and M. Ishikawa, Complete inhibition of tobamovirus multiplication by simultaneous mutations in two homologous host genes, J. Virol., 2002, 76, 2494-2497.

6 T. Yamanaka, T. Ohta, M. Takahashi, T. Meshi, R. Schmidt, C. Dean, S. Naito and M. Ishikawa, TOM1, an arabidopsis gene required for efficient multiplication of a tobamovirus, encodes a putative transmembrane protein, Proc. Natl. Acad. Sci. U. S. A., 2000, 97, 10107-10112.

7 M. S. Padmanabhan, S. P. Goregaoker, S. Golem, H. Shiferaw and J. N. Culver, Interaction of the tobacco mosaic virus replicase protein with the Aux/IAA protein PAP1/IAA26 is associated with disease development, J. Virol., 2005, 79, 2549-2558.

8 M. S. Padmanabhan, S. R. Kramer, X. Wang and J. N. Culver, Tobacco mosaic virus replicase-auxin/indole acetic acid protein interactions: reprogramming the auxin response pathway to enhance virus infection, J. Virol., 2008, 82, 2477-2485.

9 H. Ueda, Y. Yamaguchi and H. Sano, Direct interaction between the tobacco mosaic virus helicase domain and the ATP-bound resistance protein, $\mathrm{N}$ factor during the hypersensitive response in tobacco plants, Plant Mol. Biol., 2006, 61, 31-45.

10 K. Ishibashi, K. Masuda, S. Naito, T. Meshi and M. Ishikawa, An inhibitor of viral RNA replication is encoded by a plant resistance gene, Proc. Natl. Acad. Sci. U. S. A., 2007, 104, 13833-13838.

11 M. Kato, K. Ishibashi, C. Kobayashi, M. Ishikawa and E. Katoh, Expression, purification, and functional characterization of an n-terminal fragment of the tomato mosaic virus resistance protein tm-1, Protein Expression Purif., 2013, 89, 1-6.

12 K. Ishibashi, Y. Kezuka, C. Kobayashi, M. Kato, T. Inoue, T. Nonaka, M. Ishikawa, H. Matsumura and E. Katoh, Structural basis for the recognition-evasion arms race between tomato mosaic virus and the resistance gene tm-1, Proc. Natl. Acad. Sci. U. S. A., 2014, 111, 3486-3495.

13 K. Ishibashi and M. Ishikawa, The Resistance Protein Tm-1 Inhibits Formation of a Tomato Mosaic Virus Replication Protein-Host Membrane Protein Complex, J. Virol., 2013, 87, 7933-7939.

$14 \mathrm{~K}$. Ishibashi and M. Ishikawa, Mechanisms of tomato mosaic virus RNA replication and its inhibition by the host resistance factor Tm-1, Curr. Opin. Virol., 2014, 9, 8-13.

15 M. Nishikiori, S. Sugiyama, H. Y. Xiang, M. Niiyama, K. Ishibashi, T. Inoue, M. Ishikawa, H. Matsumura and E. Katoh, Crystal structure of the superfamily 1 helicase from tomato mosaic virus, J. Virol., 2012, 86, 7565-7576.

16 H. Xiang, K. Ishibashi, M. Nishikiori, M. C. Jaudal, M. Ishikawa and E. Katoh, Expression, purification, and functional characterization of a stable helicase domain from a tomato mosaic virus replication protein, Protein Expression Purif., 2012, 81, 89-95.

17 Q. Zhou Study on molecular design, synthesis and bioactivity of insecticides based on the RNA helicase of tobacco Mosaic virus, Dissertation, Guizhou University, 2014.

18 C. Y. Zhu, Y. H. Wu, X. X. Zhao and Y. H. Wang, Action mechanism of Cytosinpeptidemycin against tobacco mosaic virus, Acta Phys. Sin., 2006, 36, 314-316.

19 C. Y. Zhu, The Action mechanism of Cytosinpeptidemycin on tobacco mosaic virus, Dissertation, Shenyang Agricultural University, 2005.

20 Y. L. Li, The Action Mechanism of Cytosinpeptidemycin on the Replication Protein Gene Expression of Tobacco Mosaic Virus, Dissertation, Shenyang Agricultural University, 2017.

21 X. Y. Li, B. A Song, X. Chen, Z. C. Wang, M. J. Zeng, D. D. Yu, D. Y. Hu, Z. Chen, L. H. Jin, S. Yang, C. G. Yang and B. E. Chen, Crystal structure of a four-layer aggregate of engineered TMV CP implies the importance of terminal residues for oligomer assembly, PLoS One, 2013, 8, e77717.

22 X. Y. Li, G. F. Hao, Q. M. Wang, Z. Chen, Y. Ding, Y. Lu, D. Y. Hu and B. A. Song, Ningnanmycin inhibits tobacco mosaic virus virulence by binding directly to its coat protein discs, Oncotarget, 2017, 8, 82446-82458.

23 W. L. DeLano, The pymol molecular graphics system 2010.

24 X. Y. Li, J. Liu, X. Yang, Y. Ding, J. Wu, D. Y. Hu and B. A. Song, Studies of binding interactions between Dufulin and southern rice black-streaked dwarf virus P9-1, Bioorg. Med. Chem., 2015, 23, 3629-3637. 
25 C. J. Wienken, P. Baaske, U. Rothbauer, D. Braun and S. Duhr, Protein-binding assays in biological liquids using microscale thermophoresis, Nat. Commun., 2010, 1, 100.

26 A. Das and G. S. Kumar, Binding studies of aristololactam- $\beta$ D-glucoside and daunomycin to human serum albumin, RSC Adv., 2014, 4, 33082-33090.

27 J. N. Ding, Y. J. Zhang, H. Zhong, C. C. Ao, J. Li and J. G. Han, An all-atom molecular dynamics study of the anti-interferon signaling of ebola virus: interaction mechanisms of ebov vp24 binding to karyopherin alpha5, Mol. BioSyst., 2017, 13, 1031-1045.

28 L. A. Bratholm and J. H. Jensen, Protein structure refinement using a quantum mechanics-based chemical shielding predictor, Chem. Sci., 2016, 8, 2061-2072.

29 F. Ntie-Kang, S. Kannan, K. Wichapong, L. C. Owono Owono, W. Sippl and E. Megnassan, Binding of pyrazole-based inhibitors to mycobacterium tuberculosis pantothenate synthetase: docking and mm-gb(pb)sa analysis, Mol. BioSyst., 2014, 10, 223-239.

30 X. Wang, Z. Kelman and J. N. Culver, Helicase ATPase activity of the tobacco mosaic virus $126-\mathrm{kDa}$ protein modulates replicase complex assembly, Virology, 2010, 402, 292-302.

31 Z. Cheng, D. Muhlrad, M. K. Lim, R. Parker and H. Song, Structural and functional insights into the human Upf1 helicase core, $E M B O$ J., 2007, 26, 253-264.

32 S. S. Velankar, P. Soultanas, M. S. Dillingham, H. S. Subramanya and D. B. Wigley, Crystal structures of complexes of PcrA DNA helicase with a DNA substrate indicate an inchworm mechanism, Cell, 1999, 97, 75-84.

33 H. Xiang, S. Sugiyama, H. Adachi, K. Takano, S. Murakami, T. Inoue, Y. Mor, M. Ishikawa and E. Katoh, Crystallization and preliminary X-ray crystallographic analysis of a helicase-like domain from a tomato mosaic virus replication protein, Acta Crystallogr., Sect. F: Struct. Biol. Cryst. Commun., 2011, 67, 1649-1652.

34 C. H. Gross and S. Shuman, Mutational analysis of vaccinia virus nucleoside triphosphate phosphohydrolase II, a DExH box RNA helicase, J. Virol., 1995, 69, 4727-4736.

35 A. Pause and N. Sonenberg, Mutational analysis of a DEAD box RNA helicase: the mammalian translation initiation factor eIF-4A, EMBO J., 1992, 11, 2643-2654. 